\title{
Study of Para-Phenylenediamine (PPD) in the Clothes Textile Materials
}

\author{
Amelia Prisdiani ${ }^{1}$, Farida Tabri ${ }^{1}$, Sri Vitayani Muchtar $^{1}$, Burhanuddin Bahar ${ }^{2}$, \\ Yohanes Widodo Wirohadidjojo ${ }^{3}$, Agussalim Bukhari ${ }^{4}$ \\ ${ }^{1}$ Department of Dermatology and Venereology, Medical Faculty, Hasanuddin University, Makassar, Indonesia \\ ${ }^{2}$ Department of Biostatistic, Faculty of Public Health, Hasanuddin University, Makassar, Indonesia \\ ${ }^{3}$ Department of Dermatology and Venereology, Medical Faculty, Gajah Mada University, Yogyakarta, Indonesia \\ ${ }^{4}$ Department of Clinical Nutrition, Medical Faculty, Hasanuddin University, Makassar, Indonesia
}

\section{Email address:}

dramelia_06@yahoo.co.id (A. Prisdiani)

\section{To cite this article:}

Amelia Prisdiani, Farida Tabri, Sri Vitayani Muchtar, Burhanuddin Bahar, Yohanes Widodo Wirohadidjojo, Agussalim Bukhari. Study of Para-Phenylenediamine (PPD) in the Clothes Textile Materials. American Journal of Clinical and Experimental Medicine.

Vol. 5, No. 2, 2017, pp. 36-40. doi: 10.11648/j.ajcem.20170502.12

Received: January 26, 2017; Accepted: February 10, 2017; Published: March 1, 2017

\begin{abstract}
Atopic dermatitis is a chronic skin disease that most commonly occurs in children. The research aimed at investigating the para-phenylenenediamine (PPD) content on the textile material of the disperse blue 106 which was given the softening agent (SA). The research was conducted Biopharmaco-Laboratory of the Research Activity Centre Building, Hasanuddin University, Makassar as the place of the UV-VIS Spectrophotometer examination. The research used the experimental method to find out PPD content in the clothes textile material soaked in SA. The research result indicates that PPD content in the clothes textile material using SA is higher than the aquadest, and there is PPD increase content released by $\mathrm{SA}$ in line with the textile material soaking duration.
\end{abstract}

Keywords: Para-phenylenenediamine, Softening Agent, UV-VIS Spectrophotometer

\section{Introduction}

Atopic dermatitis (AD) is a chronic skin disease that is most common in children, typically patients less than two years old of age. Dominance in pre-school age boys and adult women had been documented [1].

The prevalence up to $12 \%$ in children aged 11 years, in research at Global International Study of Asthma and Allergies in Childhood, ranging from $1 \%$ to $20 \%$, with the highest prevalence found in Northern Europe. In $45 \%$ of children, the incidence of $\mathrm{AD}$ occurred in the first 6 months of life, while in the first year of life as much as $60 \%$, and before the age of 5 years, at least $85 \%$ of the individuals affected. In children with onset before the age of 2 years, as many as $20 \%$ will have a permanent manifestation of the AD, and in addition, as many as $17 \%$ will have intermittent symptoms at age 7 years. In adults with $\mathrm{AD}$, only $16.8 \%$ had onset after puberty. [2]

Several studies have measured morbidity AD, where AD cases often achieve the highest morbidity score compared to other diseases. In addition, a decrease in quality of life is directly related to the severity of the AD. Psychological morbidity associated with continuous scratching, disturbed sleep, and traces are visible on the skin can also affect patients. [3]

In patients with $\mathrm{AD}$ a state of hypersensitivity to external factors such as the materials used in clothes made of wool, nylon, polyester, which if exposed again will give a reaction, first response occurs early phase (early phase) followed by the slow phase (late phase reaction), Macroscopic picture seen in the slow phase is diffuse edema, erythema, and induration bit scratchy. [4]

Jeans is a type of clothing made of denim blue. It has been long used for a variety of clothing such as pants, overalls and jackets. Originally designed for cowboys and miners, then jeans became popular in the 1950s among adolescents. Became popularly used by hippies in the 1970s and in the 1980 s popular among the youth on punk rock and heavy 
metal. In the 2010s, jeans made clothing are very popular all over the world. Although jeans are known as fashion apparel popular for decades, but is still used as protective clothing by some individuals, such as farm workers and bikers, because of higher durability compared to other fabric materials. [5, 6]

Swimsuits are garments designed to be used in waterbased activities or sports, such as swimming, diving and surfing, or sunbathing. Swimsuits are used in age ranging from children to adults, both men and women. Swimsuits are made of various materials such as nylon, latex, lycra and spandex. [7]

Disperse dyes are used to color synthetic fibers. These dyes are not attached to the cloth well, and with a relatively large amount of the dye capable apart from fabric and absorbed into the skin. Disperse blue dyes 106 and 124 has been marketed since 1985 and was first reported as a cause of allergic contact dermatitis in the same year. [8]

Jeans and bathing suits are made of synthetic textile using disperse dye and physical activity causes increased production of sweat and friction, resulting in the risk of sensitization and elicitation of Contact Allergy Dermatitis. [9]

Softening agent (SA) is an ingredient in detergents containing cationic surfactants. The detergent will turn into positively charged particles when dissolved in water, typically used as a softener. [10]

One of the active ingredients contained in a softening agent is Quaternary ammonium compounds (QACs) which is an organic chemical group with a nitrogen atom in the middle, and has a branched chain group of alkali on the one hand, and on the other side of the chain metyl group or benzyl group. QACs are often used in everyday life, especially in the health sector or industry. More specifically QACs are contained surfactants in products such as fabric softeners, disinfectants, detergents, etc. QACs works as a cationic surfactant which, when ionized in water / solvent to form positive ions that serve as fabric softener. [11]

Several studies have reported that QACs can trigger contact dermatitis and bronchial asthma attacks. In addition if there is direct contact with the QACs mucous membrane and eye irritation can cause sores, irritation where heavy or mild depending on the time of exposure and concentration of QACs that cause permanent damage to the cornea. [12]

A study conducted in North America explained that QACs are the allergens that most commonly cause contact dermatitis, or approximately $16.5 \%$ of all allergens. Likewise, studies conducted in Switzerland explaining about $5.5 \%$ of all contact dermatitis patients have high sensitivity to QACs. [13]

Para-phenylenediamine (PPD) is an aromatic amine compound; with the chemical formula $\mathrm{C} 6 \mathrm{H} 8 \mathrm{~N} 2$ and its molecular weight is $108.15 \mathrm{~g} / \mathrm{mol}$. The color white to light purple powder which becomes darker when exposed to air exposure (when oxidized first - first turn red, then brown and finally to black); and slightly soluble in water. In addition to hair dye, PPD can be found on fur dyes or dye. PPD is also used as an agent for photography and antioxidants in a rubbery material. According to the Scientific Committee on Consumer Products (SCCP), PPD is a skin sensitizer which is potentially very powerful for the textile dye dermatitis. [14]

PPD include as a contact allergen according to The Centers for Disease Control and Prevention (CDC). Exposure route through inhalation, skin absorbtion, intestinal absorbtion, skin/eye contact can cause throat irritation (pharyng/laryng) asthma bronchiale and dermatitis sensititation. [14]

Measurement of levels of para-phenylenediamine (PPD) on textile materials disperse blue 106 given softening agent can be done by using a UV-VIS Spectrophotometer which can be measured the amount of light absorbed by a solution of a compound or sample. A solution of the samples are given in the maximum wavelength of light, then the light is partially absorbed, partly reflected and the rest is forwarded. Light absorbed by the sample referred to as sudirman sample. The amount of light will be recorded by a tape recorder and amplified by an amplifier contained in the UVVIS Spectrophotometer instrument that will be read on a computer as an uptake or absorbance, then by the use of an equation, the number of absorbance is calculated to be the levels of PPD in percentage. [15, 16]

Until now, the effect of softening agent on textile materials disperse blue 106 on the release of para-phenylenediamine (PPD) is unknown. Under these conditions, this study aims to determine the levels of para-phenylenediamine (PPD) on textile materials disperse blue 106 by softening agent (SA).

\section{Materials and Methods}

\subsection{Location and Time Research}

The research was conducted Biopharmaco-Laboratory of the Research Activity Centre Building, Hasanuddin University, Makassar. When the study began in November 2016.

\subsection{Design and Research Variables}

This research used experimental method using distilled water control. The research variables consist of: independent variables (duration and type of textile disperse blue 106 soaked in a softening agent), and the dependent variable (PPD levels).

\subsection{Research Samples}

Samples are textile materials clothing disperse blue 106 (brand Levis jeans material no. 505 blue, swimsuit brands Lasona color Tosca) and softening agent. Estimates of the samples based on Table Kruskal Wallis (Non Parametric Statistics) is as much as 25 samples.

\subsection{Method of Collecting Data}

Textiles garments disperse blue 106 are grouped into five treatments, namely: 1) soaked in distilled water for 10 minutes, 2) given the softening agent and soaked for 10 minutes, 3) given the softening agent and soaked for 30 
minutes, 4) given softening agent and soaked for 1 hour, and 5) given softening agent and soaked for 2 hours. After that examination with UV-VIS Spectrophotometer, followed by analysis of the levels of PPD and data processing.

\subsection{Data Analysis Technique}

Processing data using the program Statistical Package for Social Science (SPSS) version 22. The data have been obtained will be tested by ANOVA. The results of hypothesis testing is said to be significant if $\mathrm{p}<0.05$. The data was processed and presented in tables, graphs and narrative.

\section{Results}

Experimental studies have been conducted to determine levels of para-phenylenediamine (PPD) on textile materials clothes soaked in a softening agent (SA), using materials jeans and bathing suits, were divided into 5 groups, A. Group 1 material jeans size $3 \times 3 \mathrm{~cm}$ were soaked in distilled water for 10 minutes, the group 2 materials jeans size $3 \times 3 \mathrm{~cm}$ was soaked in distilled water given SA for 10 minutes, the group 3 material jeans size $3 \times 3 \mathrm{~cm}$ was soaked in distilled water given SA for 30 minutes, groups of four ingredients jeans $3 \mathrm{x}$ $3 \mathrm{~cm}$ was soaked in distilled water given SA for 1 hour, group 5 material jeans size $3 \times 3 \mathrm{~cm}$ were soaked in distilled water given SA for 2 hours, B. group 1 swimsuit material size $3 \times 3 \mathrm{~cm}$ were immersed in distilled water for 10 minutes, group 2: swimsuit material size $3 \times 3 \mathrm{~cm}$ were soaked in distilled water given SA for 10 minutes, the group 3 swimsuit material size $3 \times 3 \mathrm{~cm}$ was soaked in distilled water given SA for 30 minutes, group 4: swimsuit material size $3 \times 3 \mathrm{~cm}$ was soaked in distilled water given SA for 1 hour, group 5: swimsuit material size $3 \times 3 \mathrm{~cm}$ was soaked in distilled water given SA for 2 hours.

Explorative studies have been conducted to Determine levels of para-phenylenediamine (PPD) on textile materials clothes soaked in a softening agent (SA), using materials jeans and bathing suits, were divided into 5 groups, A. Group 1 material jeans size $3 \times 3 \mathrm{~cm}$ were soaked in distilled water for 10 minutes, the group 2 materials jeans size $3 \times 3 \mathrm{~cm}$ was soaked in distilled water given SA for 10 minutes, the group 3 material jeans size $3 \times 3 \mathrm{~cm}$ was soaked in distilled water given SA for 30 minutes, groups of four ingredients jeans $3 \mathrm{x}$ $3 \mathrm{~cm}$ was soaked in distilled water given SA for 1 hour, group 5 material jeans size $3 \times 3 \mathrm{~cm}$ were soaked in distilled water given SA for 2 hours, B. group 1 material swimsuit size $3 \times 3 \mathrm{~cm}$ were immersed in distilled water for 10 minutes, group 2: swimsuit material size $3 \times 3 \mathrm{~cm}$ were soaked in distilled water given SA for 10 minutes, the group 3 swimsuit material size $3 \times 3 \mathrm{~cm}$ was soaked in distilled water given SA for 30 minutes, group 4: swimsuit material size $3 \times 3 \mathrm{~cm}$ was soaked in distilled water given SA for 1 hour, group 5: swimsuit material size $3 \times 3 \mathrm{~cm}$ was soaked in distilled water given SA for 2 hours.

All treatments performed during one day. Results of the study were presented sequentially in the form of comparative levels of PPD in all treatment groups.

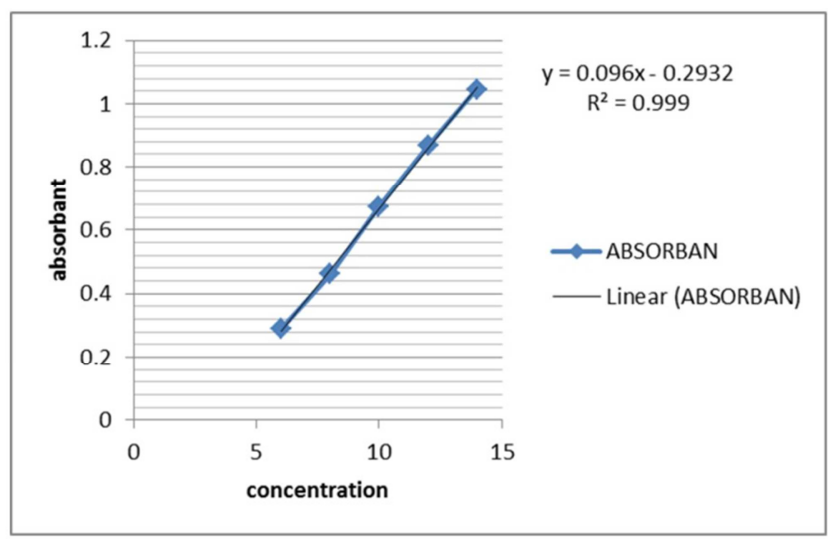

Figure 1. The standard curve.

From each group consisting of 5 samples of clothing textile materials, taken $3 \mathrm{ml}$ water sample soaked clothing textile materials to be included in the UV-VIS Spectrophotometer. Data obtained by UV-VIS Spectrophotometer checks on levels of PPD were analyzed statistically using ANOVA followed by Post Hoc analysis significant when $\mathrm{p}$ significant.

Table 1. The comparison of para-phenylenediamine (PPD) in all treatment groups.

\begin{tabular}{llll}
\hline Level of PPD & N & Mean & Std. Deviation \\
\hline Jeans without SA 10' & 5 & 3.2556 & .27348 \\
Jeans without SA 30' & 5 & 4.7596 & .40996 \\
Jeans with SA 10' & 5 & 6.8592 & .09514 \\
Jeans with SA 30', & 5 & 8.3728 & .33010 \\
Jeans with SA 1 hour & 5 & 9.0636 & .28149 \\
Jeans with SA 2 hours & 5 & 10.4956 & .13478 \\
Swimsuit without SA 10' & 5 & 2.7812 & .08579 \\
Swimsuit without SA 30' & 5 & 3.1364 & .45199 \\
Swimsuit with SA 10' & 5 & 6.4716 & .06811 \\
Swimsuit with SA 30' & 5 & 6.7960 & .11312 \\
Swimsuit with SA 1 hour & 5 & 7.1380 & .03992 \\
Swimsuit with SA 2 hours & 5 & 7.6440 & .18722 \\
\hline
\end{tabular}

Previous levels of PPD in distilled water was measured and found levels of PPD $0 \%$. From table 1 obtained the mean levels of PPD on the soaking time showed an increase in all treatment groups compared to the control group. This suggests that elevated levels of PPD is directly proportional to the duration of the soaking time.

When compared PPD jeans material without SA 10 minutes on jeans material with SA 10 minutes, PPD be twofold. When compared jeans material without SA 30 minutes on jeans material with SA 30 minutes, the amount of PPD almost 2-fold. When compared sequences between material jeans with SA 10 minutes, 30 minutes, 1 hour and 2 hours was found the increase linearly with the pattern $7,8,9,10$. Conclusion PPD while the height is associated with the use of SA and SA use that lasts longer yield levels PPD is higher.

On the substance of the swimsuit, PPD without SA 10 minutes versus PPD swimsuit with SA 10 minutes found a ratio of 1 to 2 . Between comparison PPD in a swimsuit without SA 10 minutes and 30 minutes, found 2.8 versus 3, but using SA clothes pool at 30 minutes, 2 times more than a 
swimsuit without SA 30 minutes. When the sequence is followed by administration of SA PPD time 10 minutes 2 hours was found similar to similar to the linear rise jeans material, but with an angle $\alpha$ which is smaller than the angle $\alpha$ jeans material. Conclusions while, swimsuit also generate PPD and the longer the immersion using the more PPD SA apart. PPD is apart of the swimsuit fewer than jeans material.

With a post hoc analysis, this study comparing levels of PPD in the control group to the treatment group there was significant difference $(\mathrm{p}<0.05)$ at all time of the study, in both the treatment group and the control group.

\section{Discussion}

This study shows that the levels of PPD in textiles clothing using SA higher than distilled water and there are increased levels of PPD released SA corresponding duration of immersion of textile materials.

Para-phenylenediamine (PPD) is a chemical compound most common cause of Contact Allergy Dermatitis in dermatology practice mostly known from hair dye. In addition to the hair dye, PPD is also found in hair or textile dyes. PPD is also used as an agent for photography and antioxidants in a rubbery material. According Scientic Committee on Consumer Products (SCCP), PPD is a skin sensitizer which is potentially very strong. [14] This study aims to determine the levels of PPD on textile materials clothes soaked in SA. Differences in the levels can be seen from the increase in the percentage $(\%)$ of measurement results using a UV-VIS Spectrophotometer.

Preparations SA selected because it is a mixture of alkyl chloride, dimethyl, and benzyl ammonium. This material is a sensitizer irritation, can cause allergic contact dermatitis. [10] Clothing textile materials disperse blue 106 selected for disperse blue dyes used to color synthetic fibers. These dyes are not attached to the cloth well, and with a relatively large amount of the dye capable apart from fabric and absorbed into the skin, as well as first reported as a cause of allergic contact dermatitis in the same year. [17] There on a variety of clothing, including underwear, blouses, jeans, swimwear, shoulder protectors, and velvet in leggings. [7]

The research was done quickly because according to the research objectives mentioned above have a way of research (the textile material with the treatment technique of immersion in SA) that lets obtained significant research results in a relatively short time (two hours). In this study, the reading of the result immediately after the immersion, the minutes of the $10^{\text {th }}$ and $30^{\text {th }}, 1$ hour and 2 hours after soaking.

To reduce bias in the reading results have one person trained personnel and experts in measurement of PPD using UV-VIS Spectrophotometer.

In this research, using SA soaking the clothing textile material intended to accelerate the release of PPD. From the results of initial studies to determine the release levels of PPD after soaking in SA for 10 minutes.

Can be seen in Figure 1 and Table 1, by ANOVA followed by post hoc significant difference $(\mathrm{p}<0,05)$ throughout the study period (for 10 minutes, 30 minutes, 1 hour and 2 hours) between treatment groups (textile materials garment soaked in distilled water $+\mathrm{SA}$ ) and the control group (textile materials garment soaked in distilled water).

Research shows the effect of the release of PPD more after increasing time. This shows an increase in the release of PPD after soaking in SA significantly compared with the duration of immersion so that the addition of SA in the treatment of textile materials clothing can trigger the release of PPD, which is one risk factor for contact dermatitis.

Although the literature study has been done, but have not found the information content of PPD studies of textile materials clothing can cause contact dermatitis, but another study showed levels of PPD hair dye ingredients that can cause irritant contact dermatitis of the scalp.

In this study, there are limitations which use less material variations other than denim and nylon. The use of materials disperse blue 106 on materials swimsuits soaked in SA can stimulate the release of PPD, but the levels of PPD on the material swimsuits used while swimming is not yet known and so we need other studies to determine the effect of PPD release on the content of pool water. PPD bond on materials used as examples differ from one material to the other material and the sample was not presenting any PPD at both the brand and the type of fabric.

\section{Conclusion}

From this research conclude that the levels of PPD in textiles clothing using SA is higher than the control group (distilled water). There is an increased level of paraphenylenediamine (PPD), which released the appropriate duration of immersion SA textile materials in SA.

\section{References}

[1] Siegfried, E. C., Hebert, A. A., Diagnosis of Atopic Dermatitis: Mimics, Overlaps, and Complications. J Clin Med, 2015. 4: p. 884-917.

[2] Akdis, C. A., Akdis, M., Bieber, T., Jensen, C. B., Boguniewicz, M., Eigenmann, P., et al, Diagnosis and treatment of atopic dermatitis in children and adults: European Academy of Allergology and Clinical Immunology/American Academy of Allergy, Asthma and Immunology/PRACTALL Consensus Report. Allergy, 2006, 61: p. 969-87.

[3] Katayama, I., Kohno, Y., Akiyama, K., Ikezawa, Z., Kondo, N., Tamaki, K., et al, Japanese Guideline for Atopic Dermatitis. Allergology International, 2011. 60: p. 205-220).

[4] Muitus, E. V, Risk Factors for Atopic Dermatitis. In: Bieber, T., Y. M. Leung, D., editors. Atopic dermatitis, New York: Marcel Dekker. 2002.

[5] Jeans Wikipedia [cited 2016 December 8].

[6] Ford, T. C, SNCC, Women, Denim, and the Politics of Dress. The Journal of Southern History, 2013. 79 (3): p. 625-58.

[7] Swimwear Wikipedia [cited 2016 December 8]. 
[8] Dawes-Higgs, E., Freeman, S, Allergic contact dermatitis caused by the clothing dye, disperse blue 106, an important contact allergen that may be frequently missed. Australasian Journal of Dermatology, 2004. 45: p. 64-6.

[9] Lisi, P., Stingeni, L., Cristaudo, A., Foti, C., Pigatto, P., Gola, $\mathrm{M}$, Clinical and epidemiological features of textile contact dermatitis: an Italian multicentre study. Contact Dermatitis, 2014. 70: p. 344-50.

[10] Quartenary Ammonium Compound Wikipedia [cited 2016 December 2]

[11] Xue, Y., Xiao, H., Zhang, Y, Antimicrobial Polymeric Materials with Quaternary Ammonium and Phosphonium Salts. Int J Mol Sci, 2015. 16: p. 3626-55.

[12] Gonzalez, M., Jegu, J., Kopferschmitt, M. C., Donnay, C., Hedelin, G., Matzinger, F, Asthma among workers in healthcare settings: role of disinfection with quaternary ammonium compounds. Clinical \& Experimental Allergy, 2013. 44: p. 393-406.

[13] Heederik, D. Cleaning agents and disinfectants: moving from recognition to action and prevention. Clinical \& Experimental Allergy, 2014. 44: p. 472-4.

[14] Al-Suwaidi, A., Ahmed, H, Determination of paraPhenylenediamine (PPD) in Henna in the United Arab Emirates. Int J Environ Res Public Health, 2010. 7: p. 168193.

[15] Behera, S., Ghanty, S., Ahmad, F., Santra, S., Banerjee, S, UV-Visible Spectrophotometric Method Development and Validation of Assay of Paracetamol Tablet Formulation. J Anal Bioanal Techniques, 2012.3 (6): p. 1-6.

[16] Jadhav, N. S., Lakshmi, M., M. S. M., Duraive, S, Validated Visible Spectrophotometric Estimation Of Paraphenylenediamine, A Carcinogenic Ingredient In Henna Hair Dyes. International Journal Of Pharmacy \& Technology, 2010. 2 (4): p. 900-6.

[17] Dawes-Higgs, E., Freeman, S, Allergic contact dermatitis caused by the clothing dye, disperse blue 106, an important contact allergen that may be frequently missed. Australasian Journal of Dermatology, 2004. 45: p. 64-6. 